\title{
Diagnosis of Placenta Percreta after Cesarean Delivery
}

\author{
Elizabete Pumpure*, Zane Grabe *,*, Maira Jansone*, ***, Dace Rezeberga*, ** \\ * Riga Stradins University, Riga, Latvia \\ ** Department of Gynecology, Riga East Clinical University Hospital, Riga, Latvia \\ *** Maternity Care Center, Pauls Stradins Clinical University Hospital, Riga, Latvia
}

\section{Summary}

A 30-year-old woman presented for elective cesarean section due to the suspicion of placental growth into uterine scar on ultrasound examination. At the time of the delivery morbidly adherent placenta was not detected but massive postpartum hemorrhage occurred. Patient was discharged and 13 days later emergency hysterectomy and suturing of urinary bladder was performed as a consequence of placenta percreta.

Key words: placenta percreta, placenta overlying uterine scar, repeated cesarean sections

\section{AIM OF THE DEMONSTRATION}

The incidence of placenta accrete syndrome is rising due to the growing numbers of cesarean delivery. In addition accrete syndromes have significant contribution to maternal morbidity and mortality and they are a leading cause of intractable postpartum hemorrhage and emergency peripartum hysterectomy $(5,8)$. The aim of this article is to demonstrate the value of accurate antenatal diagnosis, reasonable treatment strategy and sufficient surveillance in case of morbidly adherent placenta.

\section{CASE REPORT}

A 30-year-old woman with 2 prior cesarean sections presented at 37 weeks of gestation in a tertiary care facility for planned cesarean delivery due to suspicion of placental growth into uterine scar on ultrasound examination. A healthy, 2830-g male was delivered. The placenta was removed manually with difficulties, but visually there was no evidence of disruption of the placenta or signs of morbidly adherent placenta, the lower uterine segment was thin and atonic and postpartum hemorrhage was observed with massive amounts of blood regardless of intravenous oxytocin usage, subsequently Bakri balloon was used; despite this attempt hemorrhage persisted and was further solved with uterine artery embolization. The total blood loss during operation and embolization was $3000 \mathrm{~mL}$. Patient received transfusion with 6 units of packed red blood cells postoperatively and was discharged home with recommendations on the postoperative day 6 .

12 days later patient was admitted to gynecological ward with complains of pulling pain in the lower abdomen and raised body temperature up to $38^{\circ} \mathrm{C}$. Gynecological examination revealed slightly opened uterine cervix with small amount of sanguinary discharge and also painful, enlarged uterus corresponding 19/20 pregnancy week. Ultrasound examination demonstrated enlarged uterus with mixed echogenicity content and suspicion of placental growth into urinary bladder (Picture 1); the treatment was followed by relaparotomy, which disclosed enlarged uterus with necrotic scars and urinary bladder that was fixed to the uterus at the setting of the two prior cesarean section scars (Picture 2A, 2B). Gradual dissection of urinary bladder and uterus induced perforation at the setting of placental penetration; surgery was continued with total hysterectomy without adnexa. Two lesions in urinary bladder were sutured and suprapubic cystostomy catheter was inserted by urologists. In the postoperative period patient received transfusion with 2 units of packed red blood cells. Cystostomy catheter was evacuated after 12 days and cystography performed afterwards disclosed no extravasation of contrast material. Patient was discharged home with no complains and no urinary difficulties.

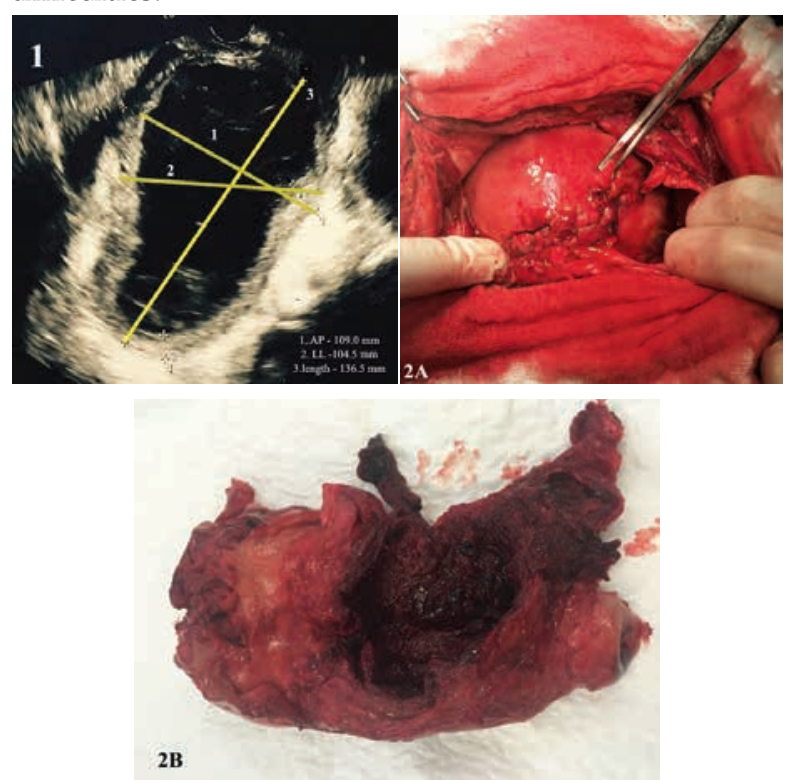

Picture 1. Ultrasound examination shows enlarged uterus (LL - 104.5mm, AP - $109.0 \mathrm{~mm}$, length $136.5 \mathrm{~mm}$ ) with mixed echogenicity content, slightly opened cavity and urinary bladder invasion. 2A. Relaparotomy reveals pale enlarged uterus with necrotic Cesarean section scar. 2B. Opened uterine cavity at the setting of necrotic Cesarean section scar and penetrating placenta 


\section{DISCUSSION}

The course of placenta percreta mostly is asymptomatic until delivery when incomplete separation of the placenta occurs and massive bleeding postpartum is the most significant sign of morbidly adherent placenta therefore the role of adequate prenatal diagnosis is becoming more important and allows time for a multidisciplinary team to make a delivery strategy $(2,4)$. Thus the ultrasound examination is a routine assessment in antenatal care and also with high sensitivity it should be the diagnostic modality of choice for placenta accreta and special attention should be paid to patients with a prior cesarean delivery and subsequent diagnosis of placenta previa or an anterior placenta overlying uterine scar. Magnetic resonance imaging should be reserved for cases in which sonography is nondiagnostic (1).

Elective hysterectomy with placenta left in situ as recommended in case of placenta accreta is not acceptable to women desiring pregnancies in future, but attempting placental removal risks hysterectomy in up to $100 \%$ of cases due to suspected massive hemorrhage (3). In described clinical case there was no suspicion of adherent placental portions, the cause of massive postpartum bleeding was thought to be atonic lower uterine segment and treatment was not continued with adequate follow-up. Follow-up of the women with retained placental portions requires serum $\beta$-hCG measurements on a weekly basis and should be supplemented by ultrasound imaging (7). Despite the use of methotrexate and embolization in case of leaving placental portions in situ have been described as a part of the conservative strategy the outcomes were the same as for receiving no additional treatment, therefore women should accept potential risks and unpredictable outcome of uterus preserving strategy and these methods should be considered experimental (6).

\section{Conflict of interest: None}

\section{REFERENCES}

1. Baughman WC, Corteville JE, Shah RR. Placenta accreta: spectrum of US and MR imaging findings // Radiographics, 2008; 28:1905-16

2. Eller AG, Bennett MA, Sharshiner M, et al. Maternal morbidity in cases of placenta accreta managed by a multidisciplinary care team compared with standard obstetric care //Obstet Gynecol, 2011; 117: 331-7

3. Eller AG, Porter TF, Soisson $\mathrm{P}$, et al. Optimal management strategies for placenta accreta // Br J Obstet Gynaecol, 2009; 1 16:648-54

4. Konijeti R, Rajfer J, Askari A.Placenta percreta and the urologist//Rev Urol, 2009;11:173-6

5. Rossi A, Lee R, Chmait R. Emergency postpartum hysterectomy for uncontrolled postpartum bleeding: a suystematic review //Obstet Gynecol, 2010; 115:637-44

6. Royal College of Obstetricians and Gynaecologists. Placenta praevia, placenta praevia accreta and vasa praevia: Diagnosis and management //Green-top guideline No. 27, 2011

7. Timmermans S, van Hof AC, Duvekot JJ Conservative management of abnormally invasive placentation //Obstet Gynecol Surv, 2007; 62: 529-39

8. Wong T: Emergency peripartum hysterectomy: a 10-year review in a tertiary obstetric hospital //N Z Med J, 2011; 124:34

\section{Address:}

Elizabete Pumpure,

Riga Stradins University,

Dzirciema Street 16,

Riga, LV-1007, Latvia

E-mail: pumpure.elizabete@gmail.com 